\title{
Effect of Spectral Power Distribution on the Resolution Enhancement in Surface Plasmon Resonance
}

\author{
Cuixia ZHOU, Guo XIA*, Guodong WANG, and Shiqun JIN \\ Laboratory of Special Display Technology of the Ministry of Education, National Engineering Laboratory of Special \\ Display Technology, National Key Laboratory of Advanced Display Technology, Academy of Photoelectric Technology, \\ Hefei University of Technology, Hefei 230009, China \\ "Corresponding author: Guo XIA_E-mail: gxia@zju.edu.cn
}

\begin{abstract}
For wavelength interrogation based surface plasmon resonance (SPR) sensors, refractive index (RI) resolution is an important parameter to evaluate the performance of the system. In this paper, we explore the influence of spectral power distribution on the refractive index (RI) resolution of the SPR system by simulating the reflectivity curve corresponding to different incident angles of the classical Kretschmann structure and several different spectral power distribution curves. A wavelength interrogation based SPR system is built, and commercial micro-spectrometers (USB2000 and USB4000) are used as the detection components, respectively. The RI resolutions of the SPR system in these two cases are measured, respectively. Both theoretical and experimental results show that the spectral power distribution has a significant effect on the RI resolution of the SPR system.
\end{abstract}

Keywords: Instrumentation; measurement; metrology; surface plasmon; resonance

Citation: Cuixia ZHOU, Guo XIA, Guodong WANG, and Shiqun JIN, "Effect of Spectral Power Distribution on the Resolution Enhancement in Surface Plasmon Resonance," Photonic Sensors, 2018, 8(4): 310-319.

\section{Introduction}

Surface plasmon resonance (SPR) is the resonant oscillation of conduction electrons at the interface between the noble metal and dielectric stimulated by the incident light [1]. Noble metals have negative permittivities such as gold and silver, and dielectric materials such as liquids, gases, or solid are used [2]. Because of the rapid enhancement of electromagnetic fields near the metal structure, SPR based optical sensors are exceedingly sensitive to small changes in the refractive index (RI) of the metal interface and have the capability of label-free real-time sensing [2].

In recent years, SPR sensors have been widely used in many fields, such as drug selection [3], clinical diagnosis [4], food detection [5], and environmental monitoring [6], which have become a standard biophysical tool [7]. According to different detection methods, SPR sensors can be divided into four types: wavelength interrogation, angle interrogation, intensity interrogation, and phase interrogation. Among them, wavelength interrogation based SPR sensors have great advantages over the other interrogation methods of SPR sensors, such as miniaturization, SPR imaging technology [8], multi-channel [9], and multi parameter measurement. However, wavelength interrogation based SPR sensors, also called spectral SPR sensors, use spectrometers as detectors, which

Received: 7 March 2018 / Revised: 9 July 2018

(C) The Author(s) 2018. This article is published with open access at Springerlink.com

DOI: $10.1007 / \mathrm{s} 13320-018-0507-8$

Article type: Regular 
largely limits their RI resolution. It is an important issue to improve the RI resolution of spectral SPR sensors. The RI resolution of sensors has the minimum change in the parameter to be determined which can be resolved by a sensing device [10]. For spectral SPR sensors, the RI resolution can be expressed as dividing the measurement accuracy of the resonance wavelength by the RI sensitivity of the sensors.

The measurement accuracy is defined as the standard deviation of multiple measurements, which is limited by the widths and the signal to noise ratio of the SPR measurement curve [11]. When the width of the SPR measurement curve increases, the uncertainty of measurement accuracy increases. Similarly, the measurement accuracy will decrease as the signal to noise ratio of the SPR measurement curve decreases. However, these two factors are directly affected by the spectral power distribution. The spectral power distribution here refers to the spectral power distribution of the system, which is related to the light source, the components of the system, and the response of the charge coupled device (CCD). On the one hand, the SPR measurement curve is also affected by the spectral power distribution and reflectivity of the SPR sensor. Once the parameters of the SPR sensor are determined (using the same SPR sensor), the width of the SPR measurement curve is only related to the spectral power distribution. On the other hand, the wavelength interrogation SPR system uses the spectrometer as the detection component. The stronger the spectral power distribution is, the higher the signal-to-noise ratio of the SPR measurement curve will be. For the RI sensitivity of the sensors, J. Homola [12] studied it in detail. The RI sensitivity is defined as the ratio of the change in the resonance wavelength to the change in the refractive index when the refractive index of the sample is changed slightly. Generally, the RI sensitivity increases with an increase in the resonant wavelength. However, the difference of spectral power distribution will lead to the different displacements of the resonance wavelength and then affect the RI sensitivity. Therefore, the RI resolution of the SPR system is mainly affected by the spectral power distribution and is different when operating at different resonant wavelengths. Furthermore, for different spectral power distributions, the optimal resonant wavelength (the optimal RI resolution) of the SPR system will also change.

The paper is organized as follows: the design of the study, the setting, the type of materials involved, a clear description of all interventions and comparisons, and the type of analysis used are given in Section 2. The noisy spectra of SPR affected by different spectral power distributions are given in Section 3. The measurement accuracy and RI sensitivity on the resonance wavelength of different spectral power distributions are studied and the optimal resonant wavelength are presented in Section 4. In Section 5, comparative experiments on USB2000 (Ocean Optics) spectrometer and USB4000 (Ocean Optics) spectrometer are presented. In the last of this paper, the results, discussion, and conclusions are given.

\section{Methods}

In this paper, the influence of the spectral power distribution on the measurement accuracy and RI sensitivity of the SPR system is investigated by the simulation model, and the influence of spectral power distribution on the resolution of RI is analyzed. The attenuated total reflection (ATR) method together with the Kretschmann configuration [13] is often used in SPR measurements. As for SPR sensor based on the prism, the configuration includes a high RI dielectric (coupled prism K9), a chrome layer with the $10 \mathrm{~nm}$ thickness, and a gold layer with the $40 \mathrm{~nm}$ thickness. As shown in Fig. 1, a light beam from the halogen lamp passes through the coupling prism. If the resonance condition is satisfied, when the evanescent wave vector matches the surface plasma 
wave vector exactly, the SPR spectrum demonstrates a dip locating at the resonance wavelength. When the refractive index of the object is changed, the condition of surface plasmon resonance is changed, and the resonant wavelength is red or blue shifted. Furthermore, the SPR system based on USB2000 (Ocean Optics) and USB4000 (Ocean Optics) is built, and the relationship between the RI resolution and the wavelength under the influence of different spectral power distributions is obtained to verify the theory. The CCD (such as Sony ILX511B and Toshiba TCD1304AP) is used as the detection element in the micro spectrometer, and their response in the $600 \mathrm{~nm}-900 \mathrm{~nm}$ band decreases. So, the spectral power distribution detected on the spectrometer shows a downward trend in this band.

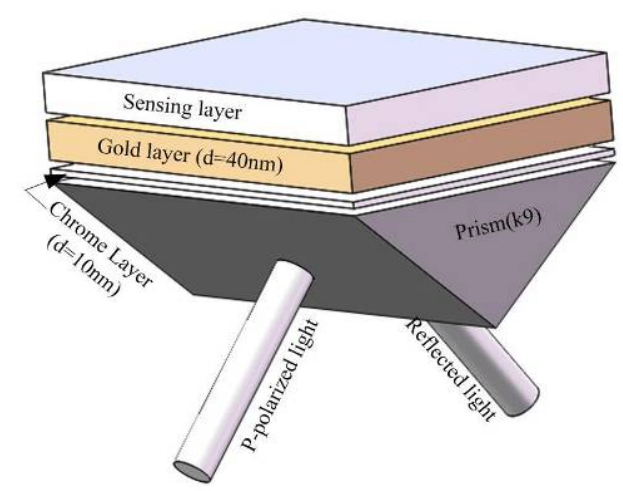

Fig. 1 SPR sensor based on the Kretschmann configuration.

The actual measured spectrum always contains noise. The noise mainly affects the measurement accuracy. The largest source of noise for the SPR sensor system is typically detector noise [15]. The noise from the spectrometer mainly can be divided into three major categories, namely readout noise, dark noise, and photoelectron noise. Because three types of noise are independent from each other, the total noise can be expressed as

$$
N=\sqrt{N_{R}^{2}+N_{D}^{2}+N_{P}^{2}}
$$

where $N$ is the total noise of the spectrometer, $N_{R}$ is the readout noise which is related to the electronic circuitry used to read the signal from CCD, $N_{D}$ is the dark noise that depends on the accumulation of dark electron and is determined by the integration time and temperature, and $N_{P}$ is the photoelectron noise which relies on the intensity of the spectrum and obeys Poisson statistics.

The SPR measurement curve is the product of the spectral power distribution and system reflectance. Considering the noise, the SPR measurement curves could be obtained as follows:

$$
S=S P D \times R_{P}+N .
$$

In addition, in terms of resonance wavelength calculation, we adopt the qualitative method. And the RI resolution curve corresponding to the USB2000 spectrometer and the USB4000 spectrometer is obtained by polynomial fitting.

\section{Modeling}

An $N$-layer structure was presented in [14] where $n_{k}$ is the complex value of the RI and $k$ is the permittivity of the $k$ th layer with a thickness $d_{k}$. The characteristic matrix of the $N$-layer structure can be expressed as

$$
\begin{aligned}
M & =\prod_{k=2}^{N-1} M_{k}=\left[\begin{array}{ll}
M_{11} & M_{12} \\
M_{21} & M_{22}
\end{array}\right] \\
& =\left[\begin{array}{cc}
\cos \beta_{k} & -\mathrm{i} \sin \beta_{k} / q_{k} \\
-\mathrm{i} q_{k} \sin \beta_{k} & \cos \beta_{k}
\end{array}\right]
\end{aligned}
$$

where $\quad \beta_{k}=\left(2 \pi d_{k} / \lambda\right)\left(\varepsilon_{k}-n_{1}^{2} \sin ^{2} \theta_{1}\right)^{1 / 2} \quad, \quad$ and $q_{k}=\left(\varepsilon_{k}-n_{1}^{2} \sin ^{2} \theta_{1}\right)^{1 / 2} / \varepsilon_{k}$. The reflection coefficient $r_{p}$ of the P-polarized (TM-polarized) incident wave can be expressed as

$$
r_{p}=\frac{\left(M_{11}+M_{12} q_{N}\right) q_{1}-\left(M_{21}+M_{22} q_{N}\right)}{\left(M_{11}+M_{12} q_{N}\right) q_{1}+\left(M_{21}+M_{22} q_{N}\right)}
$$

and therefore, the reflectance is $R_{p}=\left|r_{p}\right|^{2}$.

The typical SPR reflectivity curves at different incident angles are shown in Fig. 2. As the incident angle decreases, the SPR reflectivity curve shifts to a longer wavelength. The incidence angles are $48.5^{\circ}$, $48^{\circ}, 47.5^{\circ}, 47^{\circ}, 46.6^{\circ}, 46.1^{\circ}, 45.5^{\circ}, 44.8^{\circ}, 44^{\circ}$, and $43.1^{\circ}$, respectively.

In order to explore the influence of the spectral power distribution on the RI resolution, three different spectral power distributions are simulated, as shown in Fig. 3. 


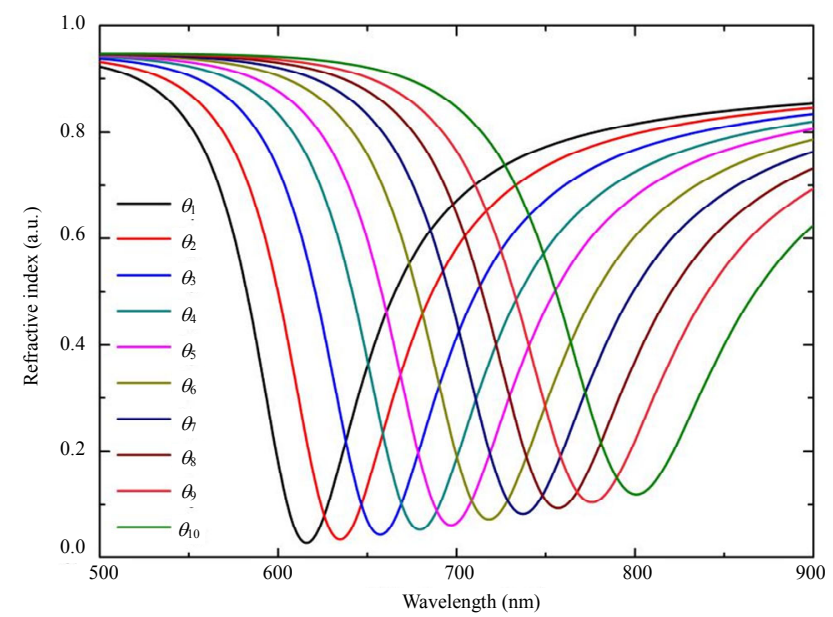

Fig. 2 Typical SPR reflectivity curves at different incident angles (the reflectivity curves from left to right correspond to incident angles $\theta_{1}, \theta_{2}, \theta_{3}, \theta_{4}, \theta_{5}, \theta_{6}, \theta_{7}, \theta_{8}, \theta_{9}$, and $\theta_{10}$, respectively).

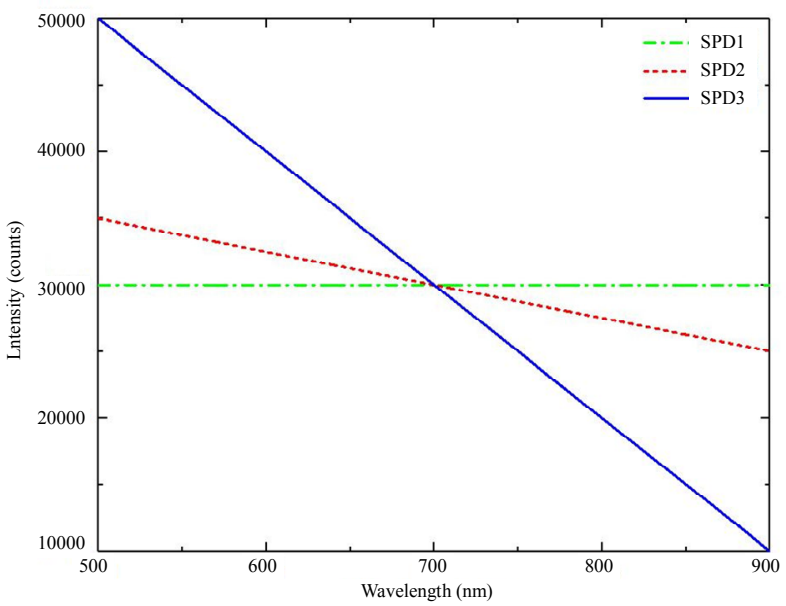

Fig. 3 Three different spectral power distributions are simulated (solid line represents SPD3, short dash line represents SPD2, and dash dot line represents SPD1).

Using the appeal formula and simulation results, different spectral power distributions are shown in the SPR measurement curves affected by three Fig. 4 to Fig. 6.

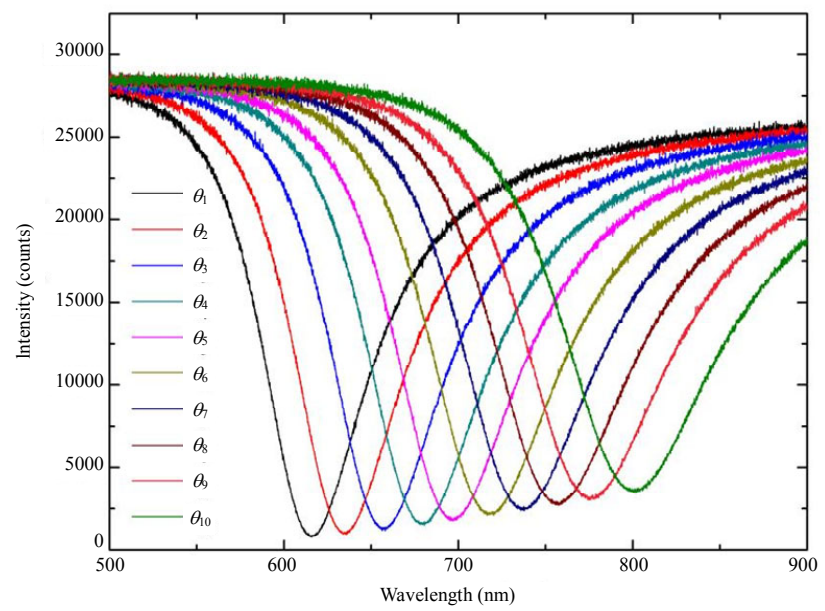

Fig. 4 SPR measurement curves affected by SPD1 (the reflectivity curves from left to right correspond to incident angles $\theta_{1}, \theta_{2}, \theta_{3}$, $\theta_{4}, \theta_{5}, \theta_{6}, \theta_{7}, \theta_{8}, \theta_{9}$, and $\theta_{10}$, respectively). 


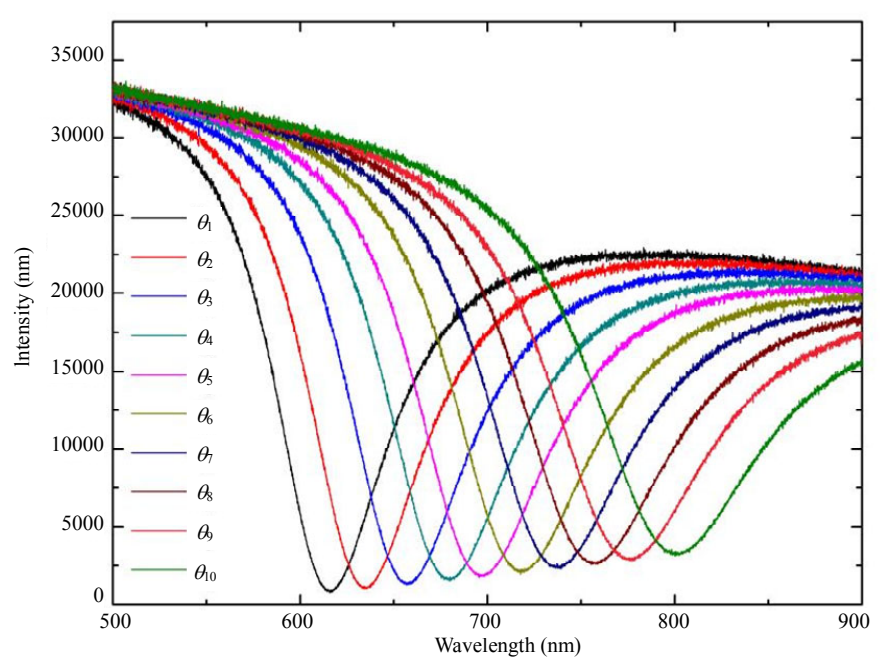

Fig. 5 SPR measurement curves affected by SPD2 (the reflectivity curves from left to right correspond to incident angles $\theta_{1}, \theta_{2}, \theta_{3}$, $\theta_{4}, \theta_{5}, \theta_{6}, \theta_{7}, \theta_{8}, \theta_{9}$, and $\theta_{10}$, respectively).

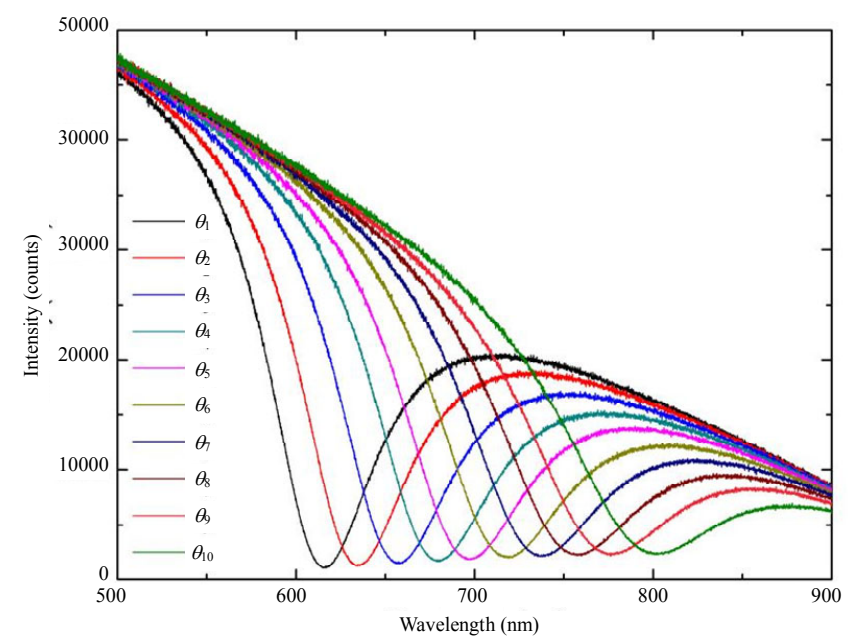

Fig. 6 SPR measurement curves affected by SPD3 (the reflectivity curves from left to right correspond to incident angles $\theta_{1}, \theta_{2}, \theta_{3}$, $\theta_{4}, \theta_{5}, \theta_{6}, \theta_{7}, \theta_{8}, \theta_{9}$, and $\theta_{10}$, respectively).

\section{Simulation}

\subsection{Measurement accuracy}

As mentioned in Section 1, the RI resolution is closely related to the measurement accuracy. The spectral width and noise of SPR will affect the measurement accuracy. When the SPR curve becomes wider or the noise level of the curve is larger, it is more difficult to measure the resonant wavelength precisely, thus reducing the measurement accuracy. However, the spectral power distribution mainly determines the width and noise level of the SPR curve. It can be seen from Fig. 4 to Fig. 6 that even with the same refractive index, the difference of the spectral power distribution forms different widths and noise levels of the SPR measurement curves.

To demonstrate this effect, we utilize the simulated SPR measurement curves affected by three different spectral power distributions which have been built in Section 3. The 1000 times SPR measurement curves are simulated, and the resonance wavelengths of each incident angle are calculated. By calculating the standard deviation of each resonance wavelength, the measurement 
accuracy of the resonant wavelength at each incident angle can be obtained. The measurement accuracy curves can be obtained by using three-order polynomial fitting, as shown in Fig. 7.

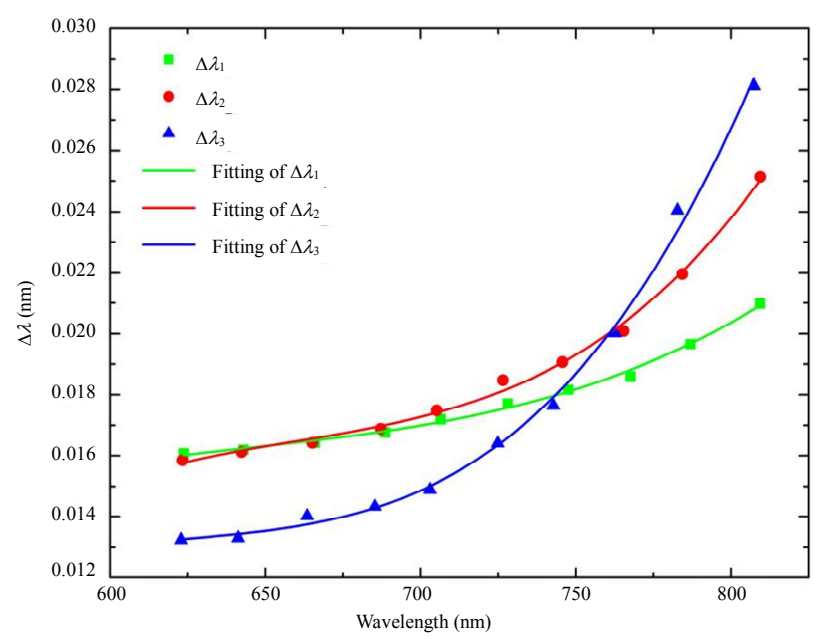

Fig. 7 Fitted curves for the measurement accuracy of the resonant wavelength.

As can be seen from Fig. 7, the standard deviations increase monotonically with the resonant wavelength, which means that the measurement accuracy decreases gradually. This is mainly because the width of the SPR curve increases as the resonant wavelength increases. Moreover, comparing the three fitting curves, we can find that the measurement accuracy of the same resonance wavelength is different due to the difference of the spectral power distribution. In the range between $500 \mathrm{~nm}$ and $700 \mathrm{~nm}$, the measurement accuracy of SPD3 is much better than that of others (the low and the better), because the corresponding SPR curve has the higher signal to noise ratio. Similarly, the measurement accuracy of SPD1 is higher than that of the other two in the range after $700 \mathrm{~nm}$ between $700 \mathrm{~nm}$ and $900 \mathrm{~nm}$.

\subsection{RI sensitivity}

A slight change in the refractive index of the analyte will cause an offset of the SPR resonant wavelength. The RI sensitivity of the SPR sensor can be defined as the ratio of the change in the resonance wavelength to the change in the refractive index of the analyte, when the refractive index of the analyte changes slightly. In order to obtain the RI sensitivity curves, the refractive index of the analyte increases from 1 to 1.000001 , and the resonance wavelength of each incident angle is calculated. Thus, the RI sensitivity of the resonance wavelength corresponding to each angle is obtained. Finally, the three-order polynomial is used to obtain the RI sensitivity curves, which represent the RI sensitivities corresponding to the resonant wavelength, as shown in Fig. 8. With an increase in the resonance wavelength, the RI sensitivity will increase accordingly. For the same resonance wavelength, the larger the slope of the spectral power distribution is, the greater the RI resolution will be.

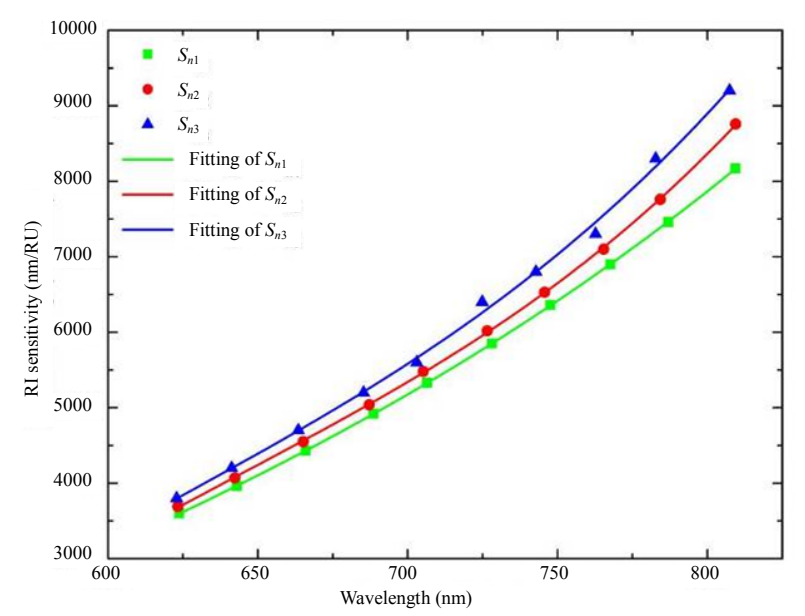

Fig. 8 Fitted curves for the RI sensitivity of the resonant wavelength .

\subsection{RI Resolution}

The RI resolution can be defined as the minimum refractive index change of an analyte that can be detected. According to the definition of Homola, the RI resolution can be represented as

$$
\delta n=\frac{\delta \lambda}{S_{n}}
$$

where $\delta n$ is the RI resolution of the sensors, $\delta \lambda$ 
is the standard deviation of the resonant wavelength, and $S_{n}$ represents the RI sensitivity of the sensors. Previously, we have obtained the measurement accuracy and RI sensitivity of the SPR system affected by three different spectral power distributions. By using (5), the RI resolution curves can be obtained, as shown in Fig. 9. We find the lowest point of each curve, which represents the best RI resolution and the corresponding resonance wavelength in this situation

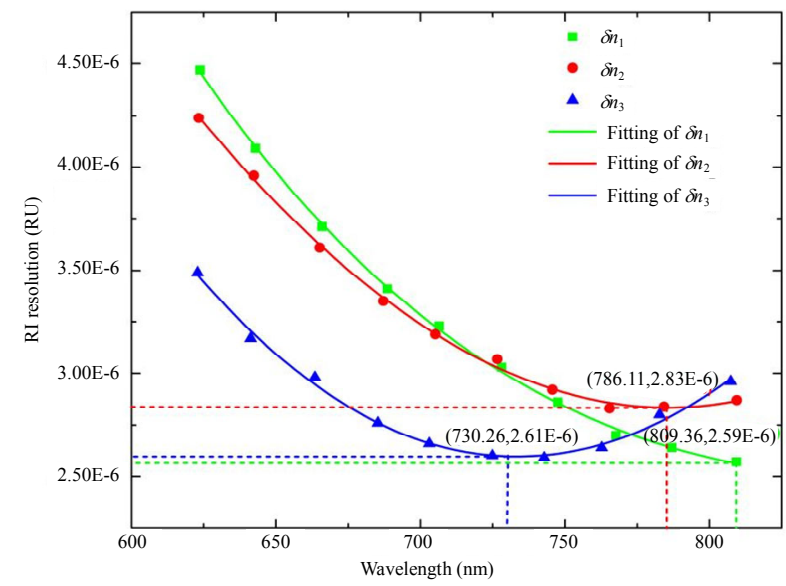

Fig. 9 Fitted curves for the RI resolution of the resonant wavelength.

It can be seen that the RI resolution of the SPD1 shows a downward trend in the full-wave band, which shows that the RI resolution will be better with an increase in the resonance wavelength. When the resonance wavelength is $786.11 \mathrm{~nm}$, the RI resolution of SPD2 is the best, whose value is $2.83 \times 10^{-6}$. Similarly, the best resonance wavelength corresponding to SPD3 is $730.2 \mathrm{~nm}$, and the resolution of the SPR system can be achieved as $2.61 \times 10^{-6}$. Compared with operating at other resonant wavelengths, the RI resolution increases. The experimental results show that even with the same measurement system, spectral power distribution will affect the RI resolution of SPR system and the position of the optimal resonant wavelength of the system.

\section{Comparative experiments based on the self-designed wavelength SPR system}

A self-designed wavelength SPR system with an adjustable incident angle is built, and its structure is shown in Fig. 10. A tungsten halogen lamp (A) is used as a light source. The light is then collimated by an optical fiber collimator (B, $\phi 5 \mathrm{~mm}$, SMA905). After being polarized by the polarizer (C, $\phi 25.4$, extinction ratio $500: 1)$, the light is incident to the surface of the SPR module (D). The SPR module is in the Kretschmann geometry: a right-angle prism (K9) coated with $10 \mathrm{~nm}$ thick chromium film on the sensor surface and $40 \mathrm{~nm}$ thick gold film on the surface of the chromium film. After the SPR phenomenon occurs on the surface of the gold film, the reflected light enters the fiber collimator (E, $\phi 5 \mathrm{~mm}$, SMA905). Finally, it is received by a $C C D$-based spectrometer $(F, G)$. The spectrometers we select are USB2000 spectrometer (F, Ocean Optics) and USB4000 spectrometer (Ocean Optics). The USB2000 spectrometer is based on the Sony ILX511B linear CCD chip which contains 2048 pixels, and the USB4000 spectrometer is based on the Toshiba TCD1304AP linear CCD chip which contains 3648 pixels. Both of them are equipped with a $25 \mu \mathrm{m}$ entrance slit, and the groove spacing is $1.667 \mu \mathrm{m} /$ line. The spectra of the tungsten halogen lamp collected by USB2000 spectrometer and USB4000 spectrometer are shown in Fig. 11. The SPR measurement curves with multiple different incident angles are shown below.

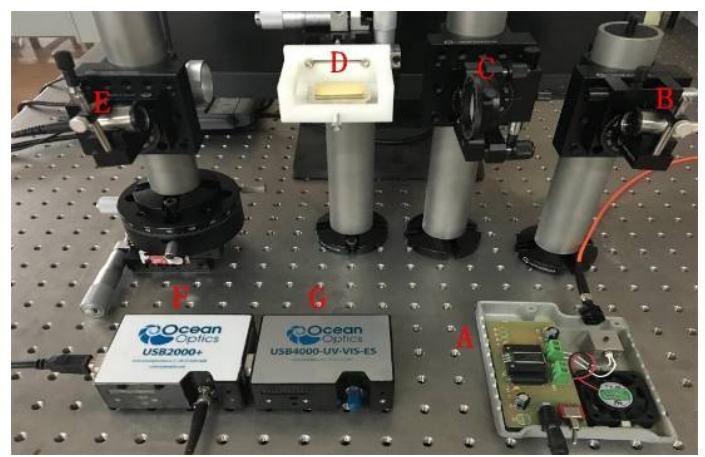

Fig. 10 A self-designed wavelength SPR system with an adjustable incident angle. 


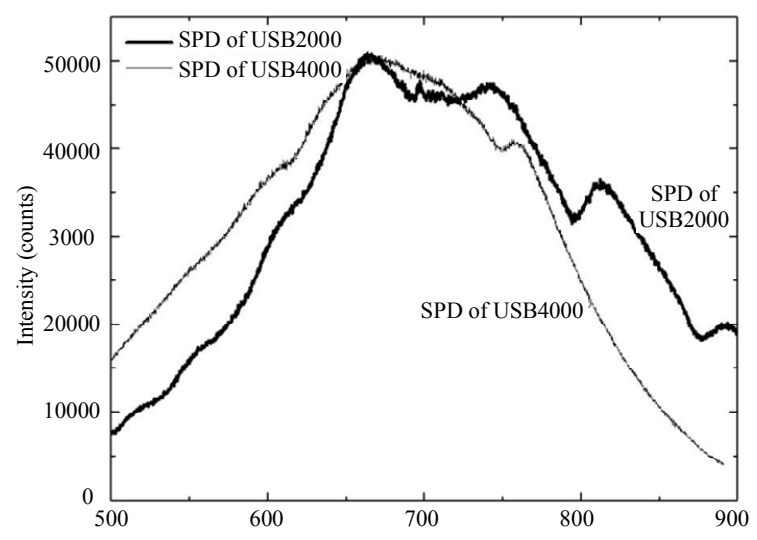

Fig. 11 Spectral power distributions collected by USB2000 spectrometer and USB4000 spectrometer.

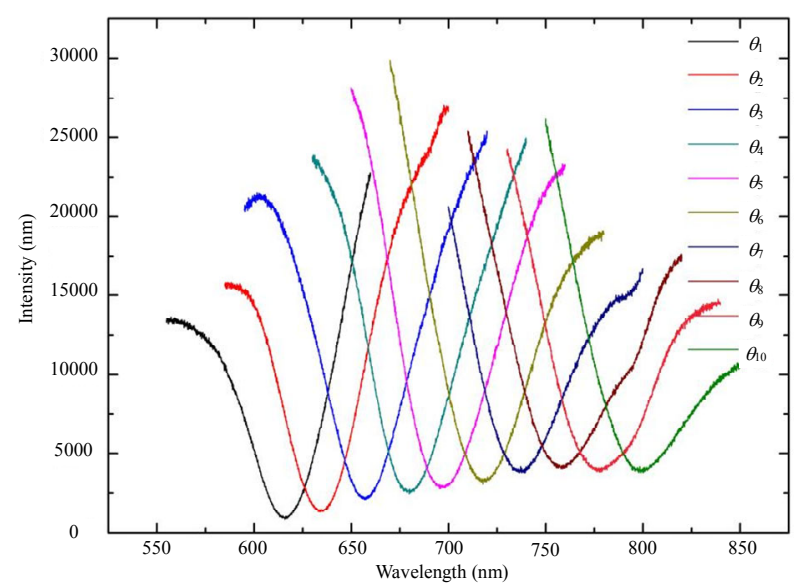

Fig. 12 SPR measurement curves acquired by USB2000 spectrometer (the reflectivity curves from left to right correspond to incident angles $\theta_{1}, \theta_{2}, \theta_{3}, \theta_{4}, \theta_{5}, \theta_{6}, \theta_{7}, \theta_{8}, \theta_{9}$, and $\theta_{10}$,respectively).

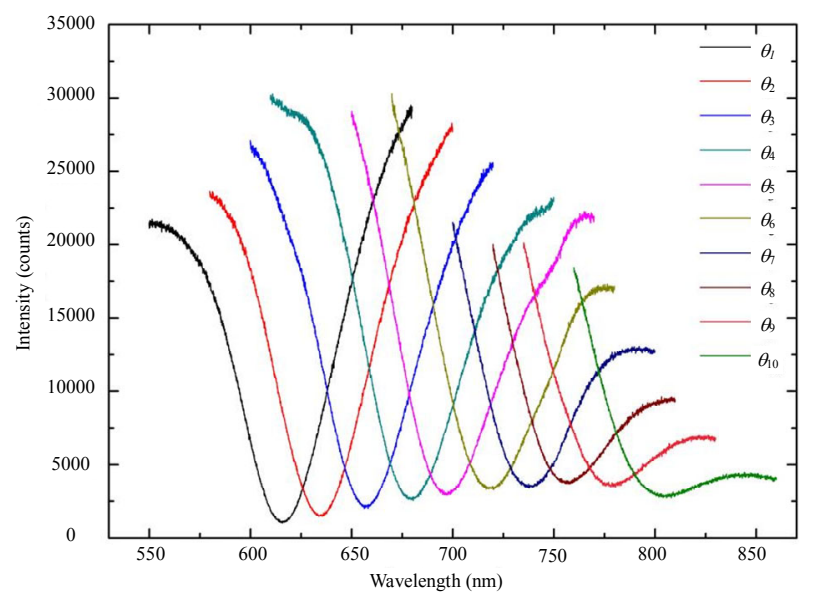

Fig. 13 SPR measurement curves acquired by USB4000 spectrometer (the reflectivity curves from left to right correspond to incident angles $\theta_{1}, \theta_{2}, \theta_{3}, \theta_{4}, \theta_{5}, \theta_{6}, \theta_{7}, \theta_{8}, \theta_{9}$, and $\theta_{10}$, respectively).
By changing the incident angle, from $\theta_{1}$ to $\theta_{10}$, the resonance wavelength changes, and the SPR measurement curves acquired through USB2000 spectrometer and USB4000 spectrometer are shown in Figs. 12 and 13, respectively. To ensure the stability of the experimental environment, the maximum measurement value of the tungsten halogen lamp spectrum is approximately kept at 50000 counts in the whole process.

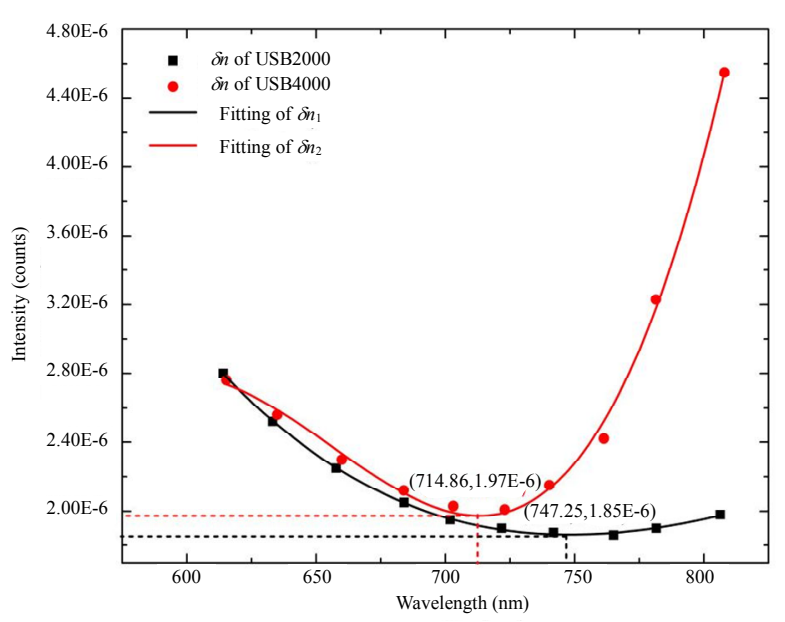

Fig. 14 RI resolution curves corresponding to USB2000 spectrometer and USB4000 spectrometer.

We measure it for 1000 times and calculate the standard deviation of each resonance wavelength. The measurement accuracy of the resonant wavelength at each incident angle can be obtained. The refractive index of the analyte changes slightly several times, and the changes in the resonance wavelength are recorded. By linear fitting, the RI sensitivity of each incident angle can be calculated. Then, the RI resolution of the resonant wavelength corresponding to the incident angles is obtained. By three-order polynomial fitting, the RI resolution curves corresponding to USB2000 spectrometer and USB4000 spectrometer are shown in Fig. 14, which shows that the RI resolutions of these two situations are different and the optimal resonance wavelengths of USB2000 spectrometer and USB4000 spectrometer for this SPR system are $747.25 \mathrm{~nm}$ and $714.86 \mathrm{~nm}$, respectively. 


\section{Results}

We have explored the effect of the spectral power distribution on the RI resolution. The RI resolution is different when the spectral power distribution of the system is different. It can be seen from Fig. 14 that the RI resolution of USB2000 spectrometer is better than that of USB4000 spectrometer. The best RI resolution of USB4000 spectrometer is $1.97 \times 10^{-6}$ when the resonance wavelength is $714.86 \mathrm{~nm}$, and the best RI resolution of USB2000 spectrometer is $1.85 \times 10^{-6}$ when the resonance wavelength is $747.25 \mathrm{~nm}$. This is because the spectral power distribution of the USB2000 spectrometer is much flatter than that of USB4000 spectrometer and has a higher spectral response. And in the range of decrease, it is similar with the situations of SPD2 and SPD3 in Section 4. The main reason for this phenomenon is that the two spectrometers use different CCDs. From 1000 experiments and the error theory, it can be known that the confidence interval of the optimum resonance wavelength of USB2000 spectrometer is $746.08 \mathrm{~nm}-748.41 \mathrm{~nm}$, and the optimum resonance wavelength of USB4000 spectrometer is $713.74 \mathrm{~nm}$ $-715.98 \mathrm{~nm}$. It is due to the difference of the spectral power distribution that the RI resolution is different. It is suitable for improving the RI resolution of a wavelength interrogation SPR system based on a micro spectrometer. It is important to note that the replacement of any component in the system may cause the inaccuracy of the experiment, so it is necessary to remeasure the system when replacing any of the components.

\section{Discussion}

The agreement between the experiment and the theory also proves that for the wavelength interrogation SPR system, the spectral power distribution of the system has a significant influence on the RI resolution of the system. It provides a reference value for the precise measurement of the wavelength interrogation SPR system. However, this method does not apply to the phase, angle, and intensity interrogation SPR systems. Moreover, the optimum resonant wavelength positions of different wavelength modulation systems are different.

\section{Conclusions}

In conclusion, we have explored the effect of the spectral power distribution on the RI resolution. We simulate the SPR measurement curves (same reflectivity curves) affected by three different spectral power distributions, calculate the measurement accuracy and RI sensitivity of each resonance wavelength, and then obtain the RI resolution curves. According to the simulated results and our analysis, the spectral power distribution affects the measurement accuracy and RI sensitivity of the SPR system and then affects its RI resolution. The agreement between the experiment and the theory also proves that for the wavelength interrogation SPR system, the spectral power distribution of the system has a significant influence on the RI resolution of the system. And on the whole, the spectral energy distribution is flatter, and the RI resolution is higher. When we use different spectrometers as detectors, we can first measure the RI resolution and the optimal resonant wavelength. Then the experiment can be set up with this condition. In this way, we can get more accurate results. This paper mainly proposes a method to improve the resolution of the wavelength modulation SPR system. The method provides a reference value for the precise measurement of the wavelength interrogation SPR system.

Open Access This article is distributed under the terms of the Creative Commons Attribution 4.0 International License (http://creativecommons.org/licenses/by/4.0/), which permits unrestricted use, distribution, and reproduction in any medium, provided you give appropriate credit to the original author(s) and the source, provide a link to the Creative Commons license, and indicate if changes were made. 


\section{References}

[1] R. H. Ritchie, "Plasma losses by fast electrons in thin films," Physical Review, 1957, 106(5): 874-881.

[2] K. N. Shushama, M. M. Rana, R. Inum, and M. B. Hossain, "Graphene coated fiber optic surface plasmon resonance biosensor for the DNA hybridization detection: simulation analysis," Optics Communications, 2017, 383: 186-190.

[3] D. R. Shankaran, K. V. Gobi, and N. Miura, "Recent advancements in surface plasmon resonance immunosensors for detection of small molecules of biomedical, food and environmental interest," Sensors \& Actuators B: Chemical, 2007, 121(1): 158-177.

[4] Y. Yanase, T. Hiragun, K. Ishii, T. Kawaguchi, T. Yanase, M. Kawai, et al., "Surface plasmon resonance for cell-based clinical diagnosis," Sensors, 2014, 14(3): 4948-4959.

[5] B. J. Yakes, J. Deeds, K. White, and S. L. Degrasse. "Evaluation of surface plasmon resonance biosensors for detection of tetrodotoxin in food matrices and comparison to analytical methods," Journal of Agricultural \& Food Chemistry, 2011, 59(3): 839-846.

[6] M. N. Weiss, R. Srivastava, H. Groger, P. Lo, and S. F. Luo, "A theoretical investigation of environmental monitoring using surface plasmon resonance waveguide sensors," Sensors \& Actuators A: Physical, 1995, 51(2-3): 211-217.

[7] Z. Salamon, H. A. Macleod, and G. Tollin, "Surface plasmon resonance spectroscopy as a tool for investigating the biochemical and biophysical properties of membrane protein systems. II: applications to biological systems," Biochimicaet Biophysica Acta (BBA) - Reviews on Biomembranes,
1997, 1331(2): 117-129.

[8] A. G. Notcovich, V. Zhuk, and S. G. Lipson, "Surface plasmon resonance phase imaging," Applied Physics Letters, 2000, 76(13): 1665-1667.

[9] M. Palumbo, C. Pearson, J. Nagel, and M. C. Petty, "A single chip multi-channel surface plasmon resonance imaging system," Sensors \& Actuators B: Chemical, 2003, 90(1): 264-270.

[10] G. Ruffato, E. Pasqualotto, A. Sonato, G. Zacco, D. Silvestri, M. Morpurgo, et al., "Implementation and testing of a compact and high-resolution sensing device based on grating-coupled surface plasmon resonance with polarization modulation," Sensors \& Actuators B: Chemical, 2013, 185(8): 179-187.

[11] M. Piliarik and J. Homola, "Surface plasmon resonance (SPR) sensors: approaching their limits," Optics Express, 2009, 17(19): 16505-16517.

[12] J. Homola, "On the sensitivity of surface plasmon resonance sensors with spectral interrogation," Sensors \& Actuators B: Chemical, 1997, 41(1): 207-211.

[13] R. Naraoka, H. Okawa, K. Hashimoto, and K. Kajikawa, "Surface plasmon resonance enhanced second-harmonic generation in Kretschmann configuration," Optics Communications, 2005, 248(1-3): 249-256.

[14] J. B. Maurya, Y. K. Prajapati, V. Singh, J. P. Saini, and R. Tripathi, "Improved performance of the surface plasmon resonance biosensor based on graphene or $\mathrm{MoS}_{2}$, using silicon," Optics Communications, 2016, 359: 426-434.

[15] G. Zonios, "Noise and stray light characterization of a compact CCD spectrophotometer used in biomedical applications," Applied Optics, 2010, 49(2): 163-169. 